\title{
The sensitivity of electrodiagnostic criteria in subtype identification at the presentation in patients of Guillain-Barre syndrome
}

\section{Mathukumalli N. ${ }^{1}$, Yaranagula S. ${ }^{2}$, A. Kanikannan M. ${ }^{3}$, Chepuru R. ${ }^{4}$, Yareeda S. ${ }^{5}$, Sarva S. ${ }^{6}$, Borgohain R. ${ }^{7}$}

DOI: https://doi.org/10.17511/ijmrr.2020.i02.01

\footnotetext{
1 Neeharika L. Mathukumalli, Assistant Professor, Department of Neurology, Nizam's Institute of Medical Sciences, Hyderabad, Telangana, India.

2 Sai Deepak Yaranagula, Senior Resident, Department of Neurology, Nizam's Institute of Medical Sciences, Hyderabad, Telangana, India.

3* Meena A. Kanikannan, Professor, Department of Neurology, Nizam's Institute of Medical Sciences, Hyderabad, Telangana, India.

${ }^{4}$ Ramesh Chepuru, Senior Resident, Department of Neurology, Nizam's Institute of Medical Sciences, Hyderabad, Telangana, India.

5 Sireesha Yareeda, Assistant Professor, Department of Neurology, Nizam's Institute of Medical Sciences, Hyderabad, Telangana, India.

6 Sailaja Sarva, Senior Lab Technologist, Department of Neurology, Nizam's Institute of Medical Sciences, Hyderabad, Telangana, India.

7 Rupam Borgohain, Professor and Head of Department, DM Neurology, Department of Neurology, Nizam's Institute of Medical Sciences, Hyderabad, Telangana, India.
}

Introduction: Electrophysiology plays a pivotal role in identifying various GBS subtypes. Purpose: This study evaluates the sensitivity of 5 known electrophysiological criteria in patients with GBS at the time of presentation. Material \& Methods: Clinical and electrophysiological data of GBS patients admitted with us between January 2011 and December 2016 were collected retrospectively from our hospital database, compiled and analyzed. Results: A total of 288 patients were included. Closer concordance was noted between the criteria in diagnosing axonal subtype (Range- $36.81 \%$ to $41.32 \%)$.Italian criteria had the highest sensitivity $(41.32 \%)$. There was a wider variation in the diagnosis of AIDP (Range- 19.79 to $34.72 \%$ ). Conclusion: As the timing of Nerve Conduction Studies (NCS) and the severity of disease influence the grouping of each patient into a specific electrophysiologic subtype, one should be cautious in interpreting electrodiagnosticdata.

Keywords: Electrophysiology in GBS, Sensitivity of electrophysiological criteria in GBS, Guillain Barre syndrome, GBS, Electrodiagnostic criteria

Corresponding Author

Meena A. Kanikannan, Professor, Department of Neurology, Nizam's Institute of Medical Sciences, Hyderabad, Telangana, India. Email: drmeenaak62@gmail.com

\section{How to Cite this Article}

Mathukumalli NL, Yaranagula SD, Kanikannan MA Chepuru R, Yareeda S, Sarva S, Borgohain R. The sensitivity of electrodiagnostic criteria in subtype identification at the presentation in patients of Guillain-Barre syndrome. Int J Med Res Rev. 2020;8(2):140-147.

Available From

https://ijmrr.medresearch.in/index.php/ijmrr/article/ view/1186

\section{To Browse}

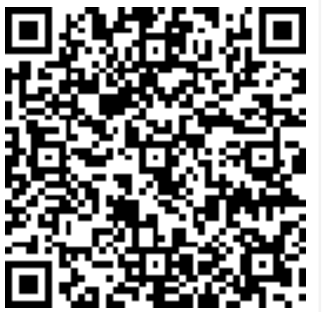

Manuscript Received 2020-02-26

Conflict of Interest No
Review Round 1 2020-03-08

Funding
Review Round 2 2020-03-13

Ethical Approval Yes
Review Round 3

Plagiarism X-checker $6 \%$
Accepted 2020-03-18

Note

(C) 2020 by Neeharika L. Mathukumalli, Sai Deepak Yaranagula, Meena A. Kanikannan, Ramesh Chepuru, Sireesha Yareeda, 


\section{Introduction}

Guillain-Barré Syndrome (GBS) is an acute inflammatory polyradiculoneuropathy. According to WHO, the overall incidence of GBS is 0.4 to 4.0 people per 1,00,000 per year [1]. Typically, the disease manifests as ascending symmetrical weakness with diminished reflexes with or without sensory involvement.Individuals of all ages can be affected, but it's more common among adults and particularly in the male.

Patients usually reach the point of greatest weakness or paralysis days or weeks after the first symptoms occur. Symptoms then stabilize at this level for a period of days, weeks, or, sometimes, months. The recovery period may be as little as a few weeks or as long as a few years.

About 30 percent of those with Guillain-Barré still have a residual weakness after 3 years. About 3 percent may suffer a relapse of muscle weakness and tingling sensations many years after the initial attack [2].

GBS is considered to be an autoimmune disease triggered by a preceding bacterial or viral infection. Campylobacter jejuni, cytomegalovirus, EpsteinBarr virus, and Mycoplasma pneumonia are commonly identified antecedent pathogens. The past infection evokes an immune response, in turn, cross-reacts with peripheral nerve components because of the sharing of cross-reactive epitopes (molecular mimicry) [3].

It can affect sensory, motor, or autonomic fibers selectively or in combination, with an axonal or demyelinating pathology.GBS can be divided into subtypes based on nerve pathology. Acute inflammatory demyelinating polyradiculoneuropathy (AIDP), Acute motor axonal neuropathy (AMAN), and Acute motor-sensory axonal neuropathy (AMSAN) are the major subtypes recognized [4].

The pathophysiology of GBS is complex. Immune reactions directed against epitopes on myelin can cause acute inflammatory demyelinating neuropathy (AIDP) [5]. The pathology is multifocal inflammatory demyelination starting at the level of the nerve roots.

The initial changes are frequently seen at the nodes of Ranvier. Both the cellular and humoral immune responses participate in the process. Invasion by activated T-cells is followed by macrophagemediated demyelination with evidence of
Complement and immunoglobulin deposition on myelin and Schwann cells [6-8].

Molecular mimicry is also the proposed pathogenetic mechanism for axonal forms of GBS. AMAN is strongly associated with antecedent Campylobacter jejuni infection, whose lipopolysaccharide capsule shares epitopes with GM1 and GD1a gangliosides concentrated at the nodes of Ranvier.

Supporting this are the early pathological findings on nerve biopsy of AMAN patients including the lengthening of the nodes of Ranvier followed by local recruitment of macrophages.

When the homologous antibody binds to nodes of Ranvier, sodium channel disruption takes place with subsequent complement activation and macrophage recruitment. Macrophages distort paranodal axons and lead to Wallerian degeneration. The inflammation in nerves of axonal forms characteristically shows the paucity of T Iymphocyte infiltration [9].

Electrophysiology plays an important role in identifying pathology. Over the decades, a number of electrophysiological criteria have been evolved for the sub typing of GBS [10-18]. Studies comparing the various criteria have been few [19-21].

This study was done with the aim of describing the clinical and electrophysiological findings in a cohort of patients with GBS and to evaluate the sensitivity of 5 electrophysiological criteria in patients with GBS at the time of presentation.

\section{Material and Methods}

Study Design: Retrospective study.

Study Period: January 2011 to December 2016

Ethical approval: Institutional ethical committee approval was obtained prior to the initiation of the study

Inclusion Criteria: Subjects who had progressive (not exceeding 4 weeks), relatively symmetrical motor flaccid weakness, involving more than one limb, and/or ataxia.

Exclusion Criteria: GBS mimics like acute peripheral neuropathies caused by HIV, Herpes simplex viruses, thiamine deficiency, very high blood sugars, toxins, Lyme's disease, tick paralysis, porphyria, vasculitic neuropathy, acute presentations of neuromuscular junction disorders, botulism, acute myositis, periodic paralysis 
Sample Size: A total of 348 case records of admitted patients were taken under the study. Of the 348 cases, 60 were excluded due to inadequate details. A total of 288 subjects were included and evaluated.

\section{Study Tools: Pre-designed pre-tested questionnaire and Keypoint Medtronic electromyography (EMG) machine (Medtronic Functional Diagnostics A/S, Skovlunde, Denmark).}

Criteria used to diagnose: Electrophysiology was performed according to conventional standard methods by a qualified senior technician trained and experienced in electromyography using a Key point Medtronic electromyography (EMG) machine (Medtronic Functional Diagnostics A/S, Skovlunde, Denmark). Nerve Conduction Studies (NCS) were done on at least 4 motors (median, ulnar, common peroneal, and posterior tibial nerves) and 3 sensories (median, ulnar and sural nerves) nerves.

CMAP distal latency (DL), motor and sensory conduction amplitudes (CMAP and SNAP), and conduction velocities (CV) were noted. Shortest $F$ response latencies (FL) were measured after 20 stimuli. Partial motor conduction block was calculated using the difference in amplitudes between stimulation sites. The skin temperature was maintained over $32^{\circ} \mathrm{C}$. The values of each variable were then compared with the upper or lower normal limits as set by our laboratory.

For each patient, 5 different criteria - Ho et al [5]; Van der Meche et al [6]; Italian GBS criteria [7]; Hadden et al [8]; Rajabally et al [9]; for the electrophysiological diagnosis of GBS were applied at presentation, and the sensitivity of these 5 criteria in the diagnosis of GBS was evaluated. The patients were classified into demyelinating or axonal variants based on the criteria.

Those patients showing features of GBS, without meeting the conditions for axonal or demyelinating subtypes with the criteria, were categorized as "Others". The final classification of each case is determined by the fulfilment of electrophysiological criteria laid down by $\mathrm{Ho}$ et al at the end of 4 weeks of illness.

Data collection methodology:The approval from Nizam'sInstitute of the medical sciences ethics committee was obtained for this study. For all the subjects the demographic information was collected. It consisted of a name, age, sex, class, address, religion, contact number, and occupation.
For all the subjects, clinical history, examination, and electrophysiology were noted. Subject's disability at admission and discharge was evaluated using the Hughes GBS disability scale [22]. Muscle power was expressed using the MRC scale and severity was assessed using the MRC sum score. [2] Nadir was defined as the highest GBS disability score attained within 4 weeks of disease onset.

Data analysis: The collected data were coded, entered into Microsoft excel worksheet, and exported to SPSS. Data were analyzed using SPSS version 21 (IBM SPSS Statistics, Somers NY, USA). Data are presented as a percentage in categories and then presented as tables and graphs. The approximate normality of the distribution was assessed. Variables following normal distribution were summarized by the mean and standard deviation (SD); the remaining variables were summarized as median (IQR). Sensitivity was defined as the proportion of all cases of GBS meeting the given criteria of interest out of the total number of cases.

\section{Results}

A total of 348 case records of admitted patients fulfilling inclusion criteria were studied. Of these, 60 were excluded from the study due to the nonavailability of adequate details and nerve conduction study records. Two hundred and eighty-eight patients were finally included in the study for further evaluation of 5 electrophysiological criteria for subtyping of GBS.

The mean age was $38.33 \pm 14.0$ years (Range- 18 to 86 years.) The majority [165/288] were in their 2nd to 4th decade of life. Male (204) outnumbered female (84) (male: female=2.4:1). The mean MRC sum score was $38.93 \pm 9.9(n=285)$ and the median was 40 . The median Hughes scale score was a median of 4 .

The mean time to nadir ( $n=284)$ was $7.34 \pm 3.8$ days with a median of 7 days. The mean time to NCS from the onset was $8.8 \pm 5.7$ days. The exact time to nadir was not available for 4 patients but was less than 28 days.

The cohort consisted of 288 patients. Closer concordance was noted between the criteria in diagnosing axonal subtype (Range- $36.81 \%$ to $41.32 \%)$. Ho and Van der Meche criteria had equal sensitivity $(40.62 \%)$. Italian criteria had the highest sensitivity $(41.32 \%)$. 
There was a wider variation in the diagnosis of AIDP (Range- 19.79 to $34.72 \%$ ). Hadden criteria showed the highest sensitivity $(34.72 \%)$ closely followed by Ho et al (34.02\%).

Italian GBS criteria showed the lowest sensitivity for AIDP (19.79\%), It also had the highest proportion of "others" group (38.89\%) closely followed by Rajabally et al (37.85\%) (Table 1 ).

Table-1: Sensitivity of diagnostic criteria for GBS ( $n=288)$.

\begin{tabular}{|l|l|l|l|}
\hline & AIDP N (\%) & Axonal N (\%) & Others N (\%) \\
\hline Ho & $98(34.02)$ & $117(40.62)$ & $73(25.36)$ \\
\hline Van der Meche/ Dutch & $76(26.39)$ & $117(40.62)$ & $95(32.99)$ \\
\hline Italian GBS & $57(19.79)$ & $119(41.32)$ & $112(38.89)$ \\
\hline Hadden & $100(34.72)$ & $106(36.81)$ & $82(28.47)$ \\
\hline Rajabally & $69(23.96)$ & $110(38.19)$ & $109(37.85)$ \\
\hline
\end{tabular}

Application of various criteria in varying weeks of presentation

1st week:147 patients (51.04\%) were analyzed in 1 st week of disease onset. Hadden criteria were most sensitive in diagnosing AIDP (30.61\%) followed by Ho criteria (29.93\%).

However, with respect to axonal GBS, Italian criteria (36.73\%) were most sensitive, followed by Ho criteria (36.05\%). All criteria closely concurred in axonal subtype (Range- $34.02 \%$ to $36.73 \%$ ) but had a wider range in diagnosing AIDP (Range$19.05 \%$ to $30.61 \%$ ) (Table 2 ).

2nd week:103(35.76\%) patients were analyzed in 2nd week. Concordance among the criteria was again better in axonal subtype (Range - 36.89\% to $41.75 \%)$. With respect to AIDP, Hadden and Ho criteria had similar sensitivity (42.72). Italian criteria had the lowest sensitivity to AIDP (29.13\%) and the highest number of patients in the "Other" category (29.12\%). (Table 2 )

23rd week:38 (13.20\%) patients were analyzed in 3rd week and beyond. The diagnosis of axonal subtype ranged from 47.3 to $57.89 \%$. Concurrence was noted among Ho, Van der Meche, and Italian criteria (57.89\%). With respect to AIDP, Rajabally and Van der Meche had equal sensitivity (18.42\%). The highest sensitivity for AIDP was noted with Hadden criteria (28.95\%) and lowest with Italian criteria $(13.16 \%)$ (Table 2 ).

Table 2: Classification of GBS using various electrodiagnostic criteria in varying weeks of presentation.

\begin{tabular}{|c|c|c|c|c|c|c|c|c|c|}
\hline \multirow[t]{2}{*}{ Criteria } & \multicolumn{3}{|c|}{$\begin{array}{c}\text { Week } 1 \text { N (\%) } \\
n=147\end{array}$} & \multicolumn{3}{|c|}{$\begin{array}{c}\text { Week } 2 \text { N (\%) } \\
n=103\end{array}$} & \multicolumn{3}{|c|}{$\begin{array}{c}\geq \text { Week } 3 \text { N (\%) } \\
n=38\end{array}$} \\
\hline & onal & AIDP & Other & xonal & ||AIDP & Other & Axonal & | AIDP & Other \\
\hline 0 & $\begin{array}{l}53 \\
(36.05\end{array}$ & $\begin{array}{l}44 \\
(29.93\end{array}$ & $\begin{array}{l}50 \\
(34.02 \\
)\end{array}$ & $\begin{array}{l}42(40 . \\
78)\end{array}$ & $\begin{array}{l}44(42 \\
72)\end{array}$ & $\begin{array}{l}17(16 . \\
50)\end{array}$ & $\begin{array}{l}22 \\
57.89\end{array}$ & $\begin{array}{l}10(26 \\
32)\end{array}$ & $\begin{array}{l}6(15 \\
9)\end{array}$ \\
\hline $\begin{array}{l}\text { Van der } \\
\text { Meche }\end{array}$ & $\begin{array}{l}52(35 . \\
37)\end{array}$ & $\begin{array}{l}31(21 . \\
09)\end{array}$ & $\begin{array}{l}64(43 . \\
54)\end{array}$ & $\begin{array}{l}43(41 . \\
75)\end{array}$ & $\begin{array}{l}38(36 \\
89)\end{array}$ & $\begin{array}{l}22(21 . \\
36)\end{array}$ & $\begin{array}{l}22(57 . \\
89)\end{array}$ & $7^{7}(18.42$ & $9(23$ \\
\hline $\begin{array}{l}\text { Italian } \\
\text { GBS }\end{array}$ & $\begin{array}{l}54(36 . \\
73)\end{array}$ & $\begin{array}{l}22(14 . \\
97)\end{array}$ & $\begin{array}{l}71(48 . \\
30)\end{array}$ & $\begin{array}{l}43(41 . \\
75)\end{array}$ & $\begin{array}{l}30(29 \\
13)\end{array}$ & $\begin{array}{l}30(29 . \\
12)\end{array}$ & $\begin{array}{l}22(57 . \\
89)\end{array}$ & & $\begin{array}{l}11(2 \\
95)\end{array}$ \\
\hline Hadden & $\begin{array}{l}50(34 . \\
02)\end{array}$ & $\mid \begin{array}{l}45(30 . \\
61)\end{array}$ & $\begin{array}{l}52(35 . \\
37)\end{array}$ & $\begin{array}{l}38(36 . \\
89)\end{array}$ & $\begin{array}{l}44(42 \\
72)\end{array}$ & $\begin{array}{l}21(20 . \\
39)\end{array}$ & $\begin{array}{l}18(47 . \\
36)\end{array}$ & $\begin{array}{l}11(28 \\
95)\end{array}$ & $\begin{array}{l}9(23 \\
9)\end{array}$ \\
\hline Rajabally & $\begin{array}{l}51(34 . \\
69)\end{array}$ & $\begin{array}{l}28(19 . \\
05)\end{array}$ & $\begin{array}{l}68(46 . \\
26)\end{array}$ & & $\begin{array}{l}34(33 \\
00)\end{array}$ & $\begin{array}{l}29(28 . \\
17)\end{array}$ & $\begin{array}{l}19(50 . \\
00)\end{array}$ & $\begin{array}{l}7(18.4 \\
2)\end{array}$ & $\begin{array}{l}12(31 \\
58)\end{array}$ \\
\hline
\end{tabular}

Electrophysiological subtypes with the week of NCS

All criteria had progressively increased sensitivity to diagnosing axonal subtype with increasing week of NCS. All criteria showed the highest sensitivity to AIDP in 2nd week of illness followed by 1 st week.

Sensitivity to diagnosing AIDP was lowest in 3rd week and beyond. The highest proportion of patients was categorized into "Others" in the 1st week of illness irrespective of the criterion applied (Figure 1).
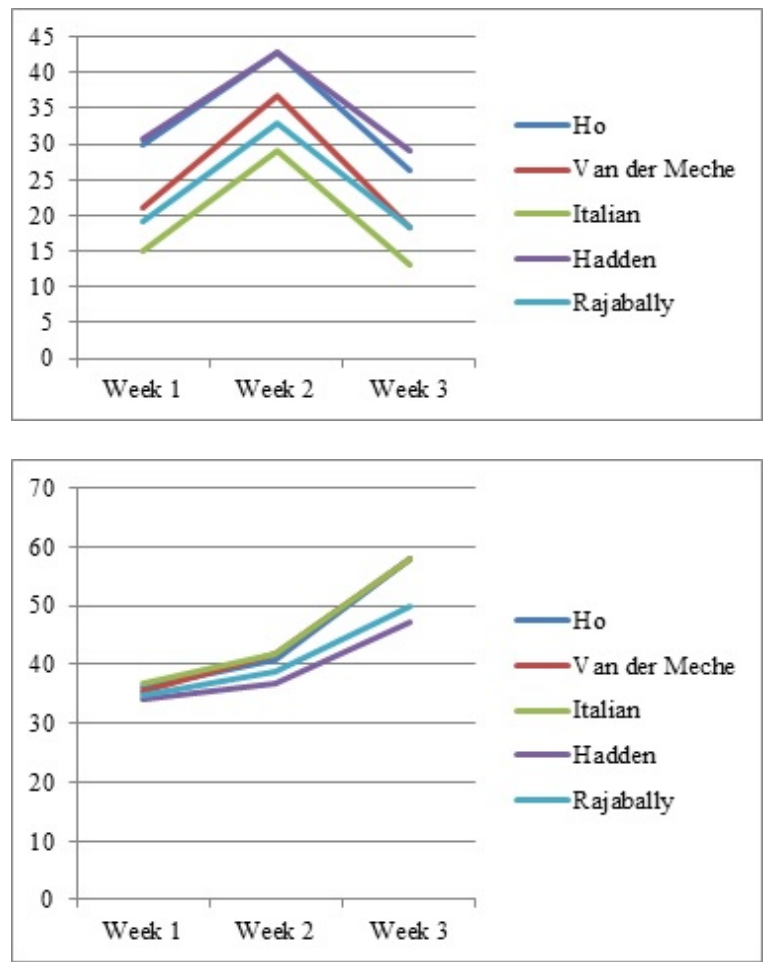


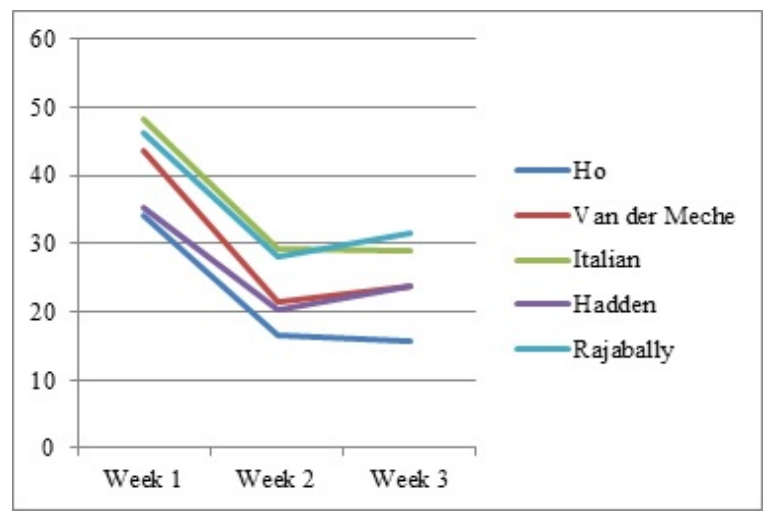

Fig-1: Trends of AIDP, axonal, and 'Others' with varying weeks of presentation, using 5 electrodiagnostic criteria.

Sural sensory conductions with GBS subtype: $A$ comparison of sural sensory conductions were done with various criteria. While SNAP amplitudes did not show any significant differences, all criteria showed significant differences when SNAP velocities were taken into consideration to differentiate between AIDP and AMAN.

There was a higher number of patients with abnormal sural SNAP velocities in the AIDP group [ $p$ values- Ho et al (0.023), Van der Meche (0.023), Italian criteria (0.012), Hadden et al (0.022), Rajabally et al (0.034)].

However, when the absent SNAPs were excluded from SNAP velocities, there was no significant difference.

The severity with GBS subtype: Patients were divided on the basis of MRC sum score (40 or less, and more than 40) and Hughes scale (3 or less, and 4 or more). In the MRC sum score 40 or less, only Italian criteria showed a significantly lower number of patients in AIDP than axonal type ( $p-0.019)$. None of the other criteria showed differences.

In the MRC sum score of more than 40, all criteria had a lesser number of patients in AIDP type [ $p$ values - Ho et al (0.138), Van der Meche, Italian criteria, and Rajabally criteria (0.0001), Hadden criteria (0.014)].

In Hughes 4 or more, Only Italian criteria showed significant differences with a lesser number of AIDP than axonal ( $p-0.006)$.

In Hughes 3 or less, all criteria had a lesser number of patients in AIDP type [p values - Ho et al (0.022), Van der Meche, Italian criteria, and Rajabally criteria (0.0001), Hadden criteria (0.057)].

\section{Discussion}

This study retrospectively analyzed the electrophysiological data in 288 patients of GBS patients at presentation. Majority of the patients in the present study were in their third decade of life, a finding similar to previous studies from India $[20,21]$ and Iran (4th decade) [3]but the contrast to other studies from Australia (6th decade) [5] and Mexico (5th decade)[23]. Men were more frequently affected similarly to that observed in most of the other studies worldwide $[6,20,21,24]$.

In this cohort, $47.7 \%$ patients had a history suggesting preceding infection, which was comparable to the Alam et al cohort (47\%) [19] and much higher than the cohort of Alexander et al (7\%) and lower than Kalita et al cohort (68.63\%) $[20,21]$. Fever is most common followed by GE and respiratory infections were similar to Kalita et al [20]. While the previous study by Alexander et al had a higher proportion of AIDP[21] this study showed an axonal variant being more common than AIDP presentation irrespective of the criteria used.

The sensitivity of 5 diagnostic criteria for electrophysiological classification was looked into. The concurrence of criteria was higher for axonal variants in all weeks of presentation than AIDP. In the diagnosis of AIDP, Ho and Hadden's criteria were most sensitive. Italian criteria were least sensitive for AIDP but most sensitive for axonal type.

The order of sensitivity in AIDP remained almost similar, irrespective of the week of presentation. All criteria showed the highest sensitivity to AIDP in the second week of presentation and lowest in the third week suggesting that the demyelinating features evolve over days peaking in the second week. With respect to axonal subtype, all criteria showed the lowest sensitivity in 1st week and highest sensitivity in 3rd week and beyond.

The highest proportion of the "others" group was found in the 1st week in all criteria (Figure 1). Taken together, these findings may suggest that the electrophysiology in 1st week may not give sufficient clue to the underlying pathology and that the changes evolve over time to show maximal demyelinating changes by the second week and are severe enough by the 3rd week to suggest an axonal degeneration irrespective of the primary pathology.

Alam et al studied 6 criteria in the diagnosis of GBS. Later studies of diagnostic criteria in GBS in the 
Indian population by Kalita et al and Alexander et al revealed important information. The three studies tested the same criteria. $[19,20,21]$.

Although the current study tested the Italian, Dutch (Van der Meche) and Ho criteria similar to the above studies, the remaining 2 criteria (Hadden and Rajabally) used in the present study were different. Alam et al, Kalita et al and Alexander et al, found Albers as most sensitive and Cornblath as least sensitive for AIDP.

The present study did not apply both these criteria. Alam et al, Kalita et al and Alexander et al found a higher incidence of AIDP when compared to the present study, with Italian, Van der Meche, and Ho criteria (Table 3 ).

This is probably due to the differences in the characteristics of the cohorts. All criteria had lower sensitivity for AIDP, in the less severe subgroup (Hughes grade 3 or less) of our cohort.

This suggests that the criteria under-report AIDP in less severely affected patients of GBS. The less severe nature of our cohort as a whole compared to previous studies may be the reason for the lower sensitivity of the criteria for AIDP.

The timing from onset to NCS was also lower in our cohort and may have contributed to the lesser sensitivity to AIDP than the other studies (Table 3). The Hadden and Rajabally criteria also showed lower sensitivity in our cohort (34.72\% and $23.96 \%$ respectively) when compared to the studies by Hadden et al (69\%) [8] and Rajabally et al (44.2 to $48 \%$ ) [17].

The present study has its own limitations. Sixty subjects did not have complete data for analysis.Patients having acute motor neuropathy with conduction block (AMNCB) were not included. Ho et al criteria used as reference criteria in the study itself have a sensitivity of $86.3 \%$ and specificity of $90 \%[20,25]$.

So, there is every chance to miss 10 to $15 \%$ of GBS cases. Data pertaining to the presence or absence of serum antiganglioside antibodies and histopathological examination of nerves which helps in verifying the validity of individual criteria are not included.

\section{Conclusion}

This study demonstrates that the timing of NCS and severity of the disease, both influence the grouping
Of the patient into demyelinating or axonal variants, irrespective of the criteria used. Therefore, the clinical picture must be taken into account, and the electrodiagnostic classification by the various criteria must be interpreted with caution.

And as the electrophysiological changes evolve over time in a given case of GBS, repeat conduction at various time points especially in the first few weeks of illness may be required to subtype and thus prognosticate.

\section{What does the study add to the existing knowledge}

The present study finding showed that the criteria under-report AIDP in less severely affected patients of GBS.

\section{Author's contribution}

All the authors participated actively during the whole study. All the authors reached out to each other in many ways like study design, data collection, and manuscript preparation.

\section{Reference}

01 . Alter M. The epidemiology of Guillain-Barre! syndrome. Ann Neurol. 1990;27(S1)S7-S12. doi: [Article] [Crossref]

02. Kleyweg RP, van der Meché FG, Schmitz PI. Interobserver agreement in the assessment of muscle strength and functional abilities in Guillain-Barré syndrome. Muscle Nerve. 1991;14(11)1103-1109. doi: [Article] [Crossref]

03. Yadegari S, Kazemi N, Nafissi S. Clinical and electrophysiological features of Guillain-Barré syndrome in Iran. J Clin Neuro sci. 2014;21(9)1554-1557.

doi: [Article] [Crossref]

04. Hughes RA, Cornblath, DR. Guillain-Barre syndrome. Lancet. 2005;366(9497)1653-1666. doi: [Article] [Crossref]

05. Blum S, Reddel S, Spies J, McCombe P. Clinical features of patients with Guillain-Barré syndrome at seven hospitals on the East Coast of Australia. J Peripher Nerv Syst. 2013;18(4)316-320. doi: [Article] [Crossref] 
06. Asbury AK, Arnason BG, Adams RD. The inflammatory lesion in idiopathic polyneuritisIts role in pathogenesis. Medicine (Baltimore). $1969 ; 48(3) 173-215$.

doi: [Article] [Crossref]

07. Hafer-Macko CE, Sheikh KA, Li CY, Ho TW, Cornblath DR, McKhann GM, et al. Immune attack on the Schwann cell surface in acute inflammatory demyelinating polyneuropathy. Ann Neurol. 1996;39(5)625-635.

doi: [Article] [Crossref]

08. Kieseier BC, Kiefer R, Gold R, Hemmer B, Willison $\mathrm{HJ}$, Hartung $\mathrm{HP}$. Advances in understanding and treatment of immunemediated disorders of the peripheral nervous system. Muscle Nerve. 2004;30(2)131-156. doi: [Article] [Crossref]

09. Yuki N. Pathogenesis of axonal Guillain-Barré syndrome- hypothesis. Muscle Nerve. $1994 ; 17(6) 680-682$.

doi: [Article] [Crossref]

10. Asbury AK, Arnason BG, Karp HR, Mc Farlin DE. Criteria for the diagnosis of Guillain-Barré syndrome. Ann Neurol. 1978;15(3)565-566. doi: [Article] [Crossref]

11. Albers JW, Donofrio PD, McGonagle TK. Sequential electrodiagnostic abnormalities in acute inflammatory demyelinating polyradiculoneuropathy. Muscle Nerve. 1985;8(6)528-539.

doi: [Article] [Crossref]

12. Cornblath DR. Electrophysiology in GuillainBarré syndrome. Ann Neurol. 1990;27;S517S520. doi: [Article] [Crossref]

13. Ho TW, Mishu B, Li CY, Gao CY, Cornblath DR, Griffin JW, Asbury AK, Blaser MJ, McKhann. GMGuillain-Barre syndrome in northern China, Relationship to Campylobacter jejuni infection and anti-glycolipid antibodies. Brain. 1995; 118(3)597-605.

[Crossref]

14. Visser $\mathrm{LH}$, van der Meche FG, van Doorn PA, Meulstee J, Jacobs BC, Oomes PG, et al. Guillain- Barre syndrome without sensory loss (acute motor neuropathy)- A subgroup with specific clinical, electrodiagnostic and laboratory features, Dutch Guillain-Barre Study Group. Brain. 1995;118(4)841-847.

[Crossref]
15. Italian Guillain-Barre. Study Group- The prognosis and main prognostic indicators of Guillain-Barre' syndrome. Brain. 1996;119(6)2053-2061.

[Crossref]

16. Hadden RD, Cornblath DR, Hughes RA, Zielasek J, Hartung HP, Toyka KV, et al. Electrophysiological classification of GuillainBarré syndrome- clinical associations and outcome, Plasma Exchange/Sandoglobulin Guillain-Barré Syndrome Trial Group. Ann Neurol. 1998;44(5)780-788.

[Article] [Crossref]

17. Rajabally $Y A$, Hiew FL, Winer JB. Influence of timing on electrodiagnosis of Guillain-Barré syndrome in the first six weeks- a retrospective study. J Neurol Sci. 2015;357(1-2)143-145. doi: [Article] [Crossref]

18. Uncini A, Ippoliti L, Shahrizaila N, Sekiguchi Y, Kuwabara S. Optimizing the electrodiagnostic accuracy in Guillain-Barré syndrome subtypesCriteria sets and sparse linear discriminant analysis. Clin Neurophysiol. 2017;128(7)11761183.

doi: [Article] [Crossref]

19. Alam TA, Chaudhry V, Cornblath DR. Electrophysiological studies in the Guillain-Barré syndrome distinguishing subtypes by published criteria. Muscle Nerve. 1998;21(10)1275-1279. doi: [Article] [Crossref]

20. Kalita J, Misra UK, Das M. Neurophysiological criteria in the diagnosis of different clinical types of Guillain-Barre syndrome. J NeurolNeurosurg Psychiatry. 2008;79(3)289-293. doi: [Article] [Crossref]

21. Alexander M, Prabhakar AT, Aaron S, Thomas M, Mathew V, Patil AK. Utility of neurophysiological criteria in Guillain Barre' syndrome subtype spectrum from a tertiary referral hospital in India. Neurol India. 2011;59(5)722-726. doi: [Article] [Crossref]

22. Hughes RA, Newsom-Davis JM, Perkin GD, Pierce JM. Controlled trial of prednisolone in acute neuropathy. Lancet. 1978;2(8093)750-53. doi: [Article] [Crossref]

23. De la O-Peña $D$, Robles-Figueroa $M$, ChávezPeña Q, Bedolla-Barajas M. Features of GuillainBarre syndrome in adults results of a university hospital. Rev Med Inst Mex Seguro Soc. 2015;53(6)678-685.

¡Crossref] 
24. Hahn AF. Guillain-Barré syndrome. Lancet. 1998; 352(9128)635-641.

doi: [Article] [Crossref]

25. Scarpino M, Lolli F, Carrai R, Lanzo G, Spalletti $M$, Barilaro $A$, et al. Diagnostic accuracy of neurophysiological criteria for early diagnosis of AIDP- A prospective study. Neurophysiol Clin. 2016;46(1)35-42.

doi: [Article] [Crossref] 